\title{
野生和近交稀有 鲫的遗传多样性*
}

\author{
王剑伟 王 伟 崔迎松** \\ (中国科学院水生生物研究所, 武汉 430072 )
}

摘 要 用 RAPD 技术对稀有 鲫近交 10 代及 3 个野生群体的遗传多样性和群体间差异进行了研究。 无论从多态位点的比例、个体间的共带率还是从多样性指数来看, 近交 10 代的遗传多样性极低。在 226 个 RAPD 位点中, 野生群体有近半数的位点是多态的, Shannon 多样性指数在 0.2911 0.3235 间, 表明 自然群体保持了较丰富的遗传多样性。近交 10 代与野生群体间遗传差异十分明显。野生群体间在 11 ～19 个位点上的表型频率存在显著差异，总遗传多样性的 $91.33 \%$ 来自群体内 $8.67 \%$ 来自于群体间。 关键词 稀有】鲫, RAPD, 遗传多样性, Shannon 指数

\section{Genetic diversity of three Gobiocypris rarus populations and one inbreeding stock/WANG Jian_Wei ,} WANG Wei , CUI Ying_Song

Abstract Genetic diversity of three Gobiocypris rarus populations and one inbreeding stock was studied using random amplified polymorphic DNA ( RAPD) method. Materials of natural populations were collected from three counties of Sichuan Province, Hanyuan (HY), Guanxian (GX) and Pengxian (PX), where water system belongs to three tributaries of the Yangtz River, Dadu River, Minjiang River and Tuojiang River system , respectively. The inbreeding stock was the tenth generation descendant of a brother_sister mating $\left(\mathrm{F}_{10}\right)$ system whose parents were collected in Hanyuan. Percentage of polymorphic sites , genetic similarity and the Shannon $\mathrm{s}$ index of phenotypic diversity were used to analyze genetic diversity. The results showed the genetic diversity of $\mathrm{F}_{10}$ was very low, whereas that of natural populations was relatively abundant. In natural populations, nearly half of the RAPD sites were polymorphic, and the average Shannon's index was 0.3235 ( HY) , 0.3075 (GX) and $0.2911(\mathrm{PX})$, respectively. Genetic similarity among individuals indicated that genetic diversity of HY and GX was higher than that of PX. Difference in genetic structure between $F_{10}$ and natural populations was fairly obvious. Although the differences on phenotype frequency were detected on 11 to 19 sites , no molecular marker was found to distinguish natural populations. Analysis on the coefficient of differentiation using Shannon's index revealed that $91.33 \%$ of total genetic diversity existed within natural populations and $8.67 \%$ among them. Treating the presence and absence of bands as two phenotypes, Chi_test $\left(\chi^{2}\right)$ and the analysis of Shannon's index was suggested to be effective approach for studying population differences.

Key words Gobiocypris rarus, RAPD, genetic diversity , Shannon's index

Author' s address Institute of Hydrobiology , Chinese Academy of Sciences , Wuhan 430072

稀有鲫（Gobiocypris rarus）是我国特有的一种小型鲤科鱼类，因具有性成熟快、易于饲 养、实验室内可周年产卵、连续产卵等特点,可作为一种理想的实验鱼(王剑伟,曹文宣， 1997 )。我们从 1990 年开始饲养与繁殖稀有 鲫, 现近交已传代至第 13 代, 并正在进行有关 实验动物化的研究。有关这种鱼的生物学特点,已有较多报道(王剑伟, 1992, 1999;王剑伟等， 1998 ;常剑波等,1995)，但关于其遗传背景的研究不多。何舜平等(1997,2000) 用 RAPD 技术 研究了低等鲤科鱼类的系统发育，探讨了稀有 鲫的系统位置;汪亚平等 (1997) 对不同组织 
和早期发育阶段 6 种同工酶分化表达方式进行了分析,但这些研究没有考虑个体间和群体间 差异。

稀有】鲫主要分布于四川省汉源县、灌县 (都江堰市) 和彭县 (彭州市) ,由于这三地分别 处于大渡河、岷江、沱江水系, 因此 3 个产地的稀有鲫可视为 3 个地理群体。初步研究表明, 3 个地理群在外部形态特征的比例性状上可能存在微小差异, 但尚不足以直观鉴别 3 群体; 而 野生群体与近交 10 代间则有较大的差异。本文应用 RAPD 技术对稀有 鲫 3 个野生群体和 近交 10 代的遗传多样性进行分析, 并探讨其群体间差异、分化等问题，以期为进一步的遗传背 景分析、品系培育、品系鉴定等实验动物化研究提供依据。

\section{1 材料与方法}

\section{1 实验用鱼}

实验用稀有鲫的数量及来源见表 1 ,所有个体均用 $60 \mathrm{ppm}$ 苯佐卡因麻醉并测量后, 用 酒精固定保存。在本文中 ,HY、GX、PX 分别表示汉源、灌县和彭县群体, $\mathrm{F}_{10}$ 表示近交 10 代。

\section{表 1 实验用鱼来源及样本数}

Table 1 Source and number of sampled fish

群 体

Population
来源

Source
样本数

No. of samples
汉源

HY

灌县

GX

彭县

PX

近交 10 代

$\mathrm{F}_{10}$
1997 年采于四川省汉源县, 大渡河水系

Collected from Hanyuan County , Sichuan Province in 1997 , belonging to

Dadu River system

1997 年采于四川省灌县, 峮江水系

Collected from Guanxian County, Sichuan Province in 1997, belonging to Minjiang River system

1997 年采于四川省彭县, 沱江水系

Collected from Pengxian County, Sichuan Province in 1997 , belonging to Tuojiang River system
30

由 1990 年采于汉源县的鱼通过兄妹交配方式得到的近交 10 代的个体

The tenth generation of brother_sister mating, whose parents were col-

lected from Hanyuan County, Sichuan Province in 1990

\subsection{DNA 模板的制备}

根据肖武汉等 (1997) 的方法略加修改。取肌肉 $100 \mathrm{mg}$ 左右置于 $1.5 \mathrm{~mL}$ 离心管中,加 STE90 (10 mmol/L NaCl $500 \mathrm{mmol} / \mathrm{L}$ Tris_HCl $200 \mathrm{mmol} / \mathrm{L}$ EDTA ,pH 9.0 ) 浸泡 24 h ; 倒去浸 泡液,加 STE80（0.1 mol/L NaCl ,50 mmol/L Tris_HCl ,1 mmol/L EDTA ,pH 8.0) $400 \mu \mathrm{L}$,匀 浆,用少量 STE80 冲洗玻璃棒使总体积为 $500 \mu \mathrm{L}$; 加 $10 \%$ 的 SDS $75 \mu \mathrm{L}$,蛋白酶 $\mathrm{K}$ (Merck 公 司 $20 \mathrm{mg} / \mathrm{mL}$ ) $15 \mu \mathrm{L}$, 在 $55^{\circ} \mathrm{C}$ 下消化至无明显固体成分 (通常需 $2 \sim 5 \mathrm{~h}$ ) ;再按常规酚抽提法 提取 DNA。将 DNA 溶于 TE (10 mmol/L Tris_HCl ,1 mmol/L EDTA ,pH 8.0) ,用凝胶电泳法检 测 DNA 模板的量 ( Sambrook et al. ,1993) ,并稀释至 $10 \sim 20 \mathrm{ng} / \mu \mathrm{L}$ 。

\subsection{RAPD 扩增及电泳}

扩增在 Thermlyne Amphiltron II 型 PCR 扩增仪上进行。反应总体积为 $25 \mu \mathrm{L}$, 其中 $\mathrm{dd}_{2} \mathrm{O}$

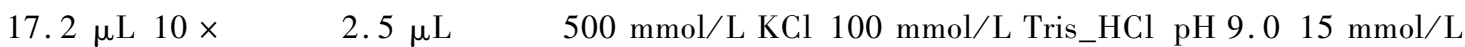
$\mathrm{MgCl}_{2} ; 1 \%$ Triton X-100), dNTPs $2 \mu \mathrm{L}$ (终浓度 $0.1 \mathrm{mmol} / \mathrm{L}$ ) ,引物 (Operon Kit J, K, 稀释为 5 $\mu \mathrm{mol} / \mathrm{L}) 1 \mu \mathrm{L}, T a q$ 酶 1/3 $\mu \mathrm{L}$ ( 1 unit, 华美) 模板 $2 \mu \mathrm{L}(10 \sim 20 \mathrm{ng} / \mu \mathrm{L})$ 。反应混和物加盖一 
层石蜡油后, 置 PCR 仪中进行反应。反应共 40 个循环, 历时约 $3 \mathrm{~h} 10 \mathrm{~min}$ 。每循环 $94^{\circ} \mathrm{C}$ 变性 $30 \mathrm{~s}, 38^{\circ} \mathrm{C}$ 复性 $30 \mathrm{~s}, 72^{\circ} \mathrm{C}$ 延伸 $90 \mathrm{~s}$ 。第一个循环前 $94^{\circ} \mathrm{C}$ 预变性 $3 \mathrm{~min}$, 最后循环后在 $72^{\circ} \mathrm{C}$ 保温 $10 \mathrm{~min}$ 。扩增产物用 $1.4 \%$ 琼脂糖凝胶电泳分离, 电压设置 $5 \mathrm{v} / \mathrm{cm}$, 采用 $\mathrm{TBE}(0.5 \times)$ 缓冲系 统, 历时约 $1 \mathrm{~h}$ 。电泳完毕, 将胶置于含溴化乙锭 $0.5 \mathrm{ug} / \mathrm{mL}$ 的 TBE 中染色 $30 \mathrm{~min}$, 取出冲洗 后在 GDS - 7600B 型 (Ultra-Violet Products Inc.) 凝胶成像系统成像。

\section{4 数据分析}

运用 $S=2 N_{x y} /\left(N_{x}+N_{y}\right)$ ，计算遗传相似率 (共有条带率) , 其中 $N_{x y}$ 表示两个体 $x 、 y$ 共有的 条带数, $N_{x}$ 及 $N_{y}$ 分别表示个体 $x 、 y$ 的条带数。由于 RAPD 标记多数是显性的 (Apostol et al. , 1993 ;Williams et al. 1990) ,且将 有带”和 无带” 视为两种表型“, 有带”为显性“, 无带”为隐 性。参照恽锐等 (1998) 的方法, 用 Shannon 信息指数计算其多样性 $: h=-\sum P_{i} \log _{2} P_{i}$, 式中 $P_{i}$ 为某位点的表型频率, 包括有带个体的频率和无带个体的频率; $h$ 为该位点的表型多样性, 它 表示群体中个体在该位点上出现 有带” 或 无带” 的不确定性。用所有位点 (包括多态位点、 单态位点) 多样性的平均值表示群体的遗传多样性。

\section{2 结果}

\subsection{RAPD 扩增的结果}

在所研究的 40 个引物中, 除 $\mathrm{OPJ}-02 、 \mathrm{OPJ}-03 、 \mathrm{OPJ}-08 、 \mathrm{OPK}-05 、 \mathrm{OPK}-18$ 无扩增带, OPJ - 09 的结果不便分析外, 其余 34 个引物均扩增出 2 到 15 条清晰可辨的条带。这些条带 分子量大都在 $0.3 \sim 2.0 \mathrm{~kb}$ 之间, 共 226 条, 平均每个引物 6.6 条 (表 2)。同一引物对不同个 体的扩增, 其结果不尽相同 (图 $1 \mathrm{~A}$ )。从 34 个引物扩增的总体结果来看, 除 $\mathrm{F}_{10}$ 的部分个体外, 野生群体中没有任何两个个体拥有完全相同的 RAPD 标记。

表 2 用于研究的 RAPD 引物及其可读的位点数

Table 2 RAPD primers and number of site researched

\begin{tabular}{|c|c|c|c|c|c|}
\hline $\begin{array}{c}\text { 引物 } \\
\text { Primer }\end{array}$ & $\begin{array}{c}\text { 序列 } \\
\text { Sequence } \\
5^{\prime} \rightarrow 3^{\prime}\end{array}$ & $\begin{array}{l}\text { 可读位点 } \\
\text { No. of site } \\
\text { researched }\end{array}$ & $\begin{array}{c}\text { 引物 } \\
\text { Primer }\end{array}$ & $\begin{array}{c}\text { 序列 } \\
\text { Sequence } \\
5^{\prime} \rightarrow 3^{\prime}\end{array}$ & $\begin{array}{l}\text { 可读位点 } \\
\text { No. of site } \\
\text { researched }\end{array}$ \\
\hline OPJ - 01 & CCCGGCATAA & 7 & OPK - 01 & CATTCGAGCC & 2 \\
\hline OPJ - 02 & CCCGTTGGGA & - & OPK - 02 & GTCTCCGCAA & 4 \\
\hline OPJ - 03 & TCTCCGCTTG & - & OPK -03 & CCAGCTTAGG & 5 \\
\hline OPJ - 04 & CCGAACACGG & 15 & OPK - 04 & CCGCCCAAAC & 8 \\
\hline OPJ -05 & CTCCATGGGG & 2 & OPK - 05 & TCTGTCGAGG & - \\
\hline OPJ - 06 & TCGTTCCGCA & 12 & OPK - 06 & CACCTTTCCC & 5 \\
\hline OPJ - 07 & CCTCTCGACA & 5 & OPK - 07 & AGCGAGCAAG & 10 \\
\hline OPJ - 08 & CATACCGTGG & - & OPK -08 & GAACACTGGG & 6 \\
\hline OPJ - 09 & TGAGCCTCAC & - & OPK - 09 & CCCTACCGAC & 10 \\
\hline OPJ - 10 & AAGCCCGAGG & 8 & OPK - 10 & GTGCAACGTG & 4 \\
\hline OPJ - 11 & ACTCCTGCGA & 2 & OPK - 11 & AATGCCCCAG & 8 \\
\hline OPJ - 12 & GTCCCGTGGT & 5 & OPK - 12 & TGGCCCTCAC & 8 \\
\hline OPJ - 13 & CCACACTACC & 6 & OPK - 13 & GGTTGTACCC & 4 \\
\hline OPJ - 14 & CACCCGGATG & 5 & OPK - 14 & CCCGCTACAC & 5 \\
\hline OPJ - 15 & TGTAGCAGGG & 6 & OPK -15 & CTCCTGCCAA & 6 \\
\hline OPJ - 16 & CTGCTTAGGG & 5 & OPK -16 & GAGCGTCGAA & 7 \\
\hline OPJ - 17 & ACGCCAGTTC & 6 & OPK -17 & CCCAGCTGTG & 6 \\
\hline OPJ - 18 & TGGTCGCAGA & 8 & OPK -18 & CCTAGTCGAG & - \\
\hline OPJ - 19 & GGACACCACT & 7 & OPK - 19 & CACAGGCGGA & 9 \\
\hline OPJ -20 & AAGCGGCCTC & 11 & $\mathrm{OPK}-20$ & FTGTCGCGAG & 9 \\
\hline
\end{tabular}






Fig. in

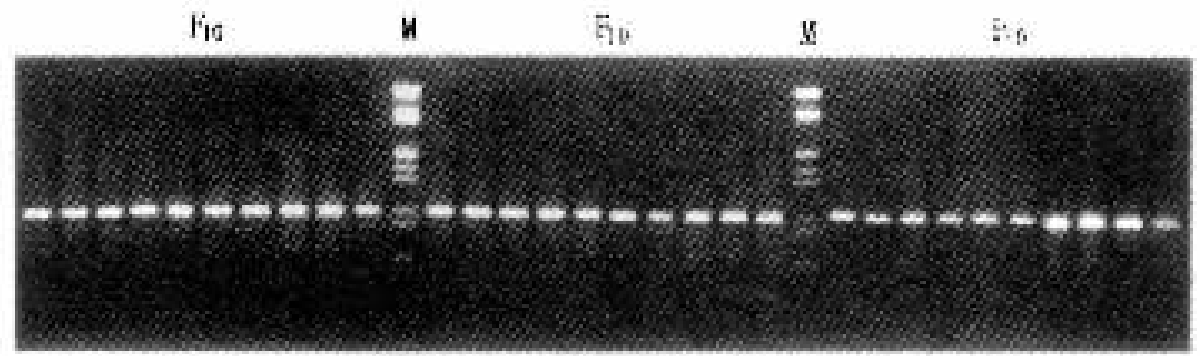

Fig. 1日

图 1 OPJ -07 引物对不同群体稀有鲫的扩增结果

Fig. 1 Electrophoretogram of PCR products of genomic DNA from different populations of Gobiocypris rarus amplified with random primer OPJ -07

箭头所指的 $500 \mathrm{bp} 、 400 \mathrm{bp}$ 带示群体间差异, M 为 $\lambda \mathrm{DNA} / H i n d \mathrm{dII}+E c o \mathrm{R} \mathrm{I}$ 分子量标准。

The bands indicated with arrows show difference between populations, M is $\lambda \mathrm{DNA} / H$ ind III + EcoR I marker.

不同群体的扩增结果也是不同的 (图 1)。在研究的 226 个位点中 ,HY 群体可检测到 224 个,, $\mathrm{GX}$ 与 PX 均为 225 个, 但 $\mathrm{F}_{10}$ 仅 201 个, 比总位点数少 25 个 (缺 25 条带), 表明 $\mathrm{F}_{10}$ 与野生群 体差异十分明显。从位点的单态性 (一个群体所有个体均有扩增带的位点) 与多态性来看, $\mathrm{HY}$ 群体有 112 个 (50\%) 多态位点, GX 有 110 个 (48.9\%),PX 有 103 个 (45.8\%)，它们之间 差别不大 ; 但 $F_{10}$ 多态位点仅 13 个, 占其位点总数的 $6.47 \%$ 。可见, $F_{10}$ 个体间具有高度的相似 性。

从野生群体缺失位点与特有位点的情况看, 虽然 HY 群体在 OPJ - 12 (1.7 k) 有一个特有 位点, 但 HY 群体只有 $60 \%$ 的个体在该位点上有扩增带。与此类似, 在 OPJ - 07 (400)、OPJ 07 (500) 这两个位点上,HY 群体缺失 (所有个体均无扩增带), 但 GX 分别只有 $33.33 \%$ 和 $43.33 \%$ 的个体有扩增带，PX 群体分别有 $16.67 \%$ 和 $43.33 \%$ 的个体有扩增带。因此，尚不能 将这些特有或缺失的位点作为鉴别野生地理群体的分子遗传标记。

\section{2 群体间遗传差异和多样性}

根据信息位点上有无扩增带, 计算出群体内个体间的遗传相似率 (表 3)。在 $95 \%$ 的显著 水平上, 群体内的遗传相似率有这样一个大小关系 : $\mathrm{F}_{10}>\mathrm{PX}>\mathrm{HY}=\mathrm{GX}$ 。其中, $\mathrm{F}_{10}$ 个体间相似 率高达 $99.622 \%$, 远远高于任何一个野生群体。相比之下, 野生群体内个体间遗传相似率低 得多，表明稀有】鲫野生群体保持了一定的遗传多样性，其中 GX 和 HY 比 PX 更丰富。

除缺失位点、特有位点外, 群体间的遗传差异还可表现在同一位点上出现扩增的个体数 (或未出现扩增的个体数) 占个体总数的百分比 (表型频率)上。对稀有】 鲫任何两个群体在 同一位点上出现、未出现扩增的个体数, 用 $2 \times 2$ 卡平方独立性检验进行分析。结果表明, $\mathrm{HY}$ 与 $\mathrm{GX}$ 间 11 个位点上的表型频率存在显著差异 $(P<0.05), \mathrm{HY}$ 与 $\mathrm{PX}$ 间 17 个、 $\mathrm{GX}$ 与 $\mathrm{PX}$ 间 
19 个位点的表型频率存在显著差异; 而 $F_{10}$ 与 HY、GX、PX 间分别有 39、46、39 个位点上存在差 异。这证明野生群体间在遗传上的确存在一定差异, 相比之下, $\mathrm{F}_{10}$ 与野生群体间的差异更大。

表 3 稀有鲫群体内遗传相似率(\%)

Table 3 Genetic similarity between individuals from the same population (\%)

\begin{tabular}{ccccc}
\hline $\begin{array}{c}\text { 群体 } \\
\text { Population }\end{array}$ & $\begin{array}{c}\text { 最小值 } \\
\text { Minimum }\end{array}$ & $\begin{array}{c}\text { 最大值 } \\
\text { Maximum }\end{array}$ & $\begin{array}{c}\text { 平均值 } \\
\text { Average }\end{array}$ & $\begin{array}{c}\text { 标准差 } \\
\text { S. D. }\end{array}$ \\
\hline $\mathrm{HY}$ & 84.270 & 96.809 & 90.306 & 2.222 \\
$\mathrm{GX}$ & 80.682 & 95.332 & 90.043 & 2.667 \\
$\mathrm{PX}$ & 82.870 & 97.409 & 91.134 & 2.540 \\
$\mathrm{~F}_{10}$ & 97.710 & 100.000 & 99.622 & 0.387 \\
\hline
\end{tabular}

遗传多样性和群体分化程度还可用 Shannon 信息指数来评价。计算结果表明 ,HY 群体平均每 个位点的多样性指数为 $0.3235, \mathrm{GX}$ 为 0.3075 ,PX 为 0.2911 , 而 $\mathrm{F}_{10}$ 仅为 0.03774 。从群体的 多样性指数可以看出, $\mathrm{F}_{10}$ 具有很低的遗传多样性, 在自然群体中, PX 的多样性较低, 这与前面 的分析结果是基本一致的。Shannon 指数还可分为群体内和群体间两部分。若将 3 个野生群 体看作一个总群体, 计算得自然群体的表型多样性为 0.3366 ,据此可计算得到自然群体的遗 传分化指数 $G s t=0.0867$, 即 $91.33 \%$ 的多样性来自于群体内, 而 $8.67 \%$ 来自于群体间, 自然群 体的遗传分化是明显的。

\section{3 讨论}

\section{1 稀有】鲫的遗传多样性和群体差异}

稀有】鲫是一种分布区较窄、生境狭小 (主要是在沟渠等小水体)、喜集群活动的小型鱼 类, 因而即使在自然环境中, 这种鱼类近亲繁殖的可能性也是较大的。本研究表明, 无论从多 态位点的比例、个体间的共带率, 还是从多样性指数来看, 近交 $\mathrm{F}_{10}$ 的遗传多样性极低; 但与近 交群不同的是, 野生群体有近半数的 RAPD 位点是多态的, 226 个位点平均表型多样度 0.3 左 右, 表明自然群体保持了较丰富的遗传多样性。这是否因选择有利于杂合个体尚有待研究。 从生态学的角度看, 稀有 鲫的生活史特点可能有助于群体保持较高的遗传多样性。稀有 鲫繁殖季节长、性成熟快、一年内能连续多次产卵(王剑伟, 1992, 1999; 王剑伟等, 1998; 常剑波 等,1995) ,当面临突然的环境变化或仔鱼面临饥饿等危险时, 也可有部分后代存活并能迅速 恢复群体数量, 保证基因不易从整个群体中丧失; 同时 环境的变迁又促使小群体的重组, 使群 体内基因得以充分交换，保证了群体基因频率的相对稳定。

本研究还表明, HY 有一个特有位点, GX 和 PX 共有 2 个, 尽管据此不能完全鉴别 3 个群 体, 但对所研究的 226 个位点表型频率的卡平方检验表明, 3 个自然群体间有 $11 \sim 19$ 个位点 上的表型频率存在显著差异。遗传多样性分析表明,91.33\% 的多样性来自于群体内, 而 $8.67 \%$ 来自于群体间。应该说, RAPD 研究的结果与形态特征研究的结果是相似的, 稀有 鲫 的确存在彼此间有一定差异的 3 个地理群体。

\subsection{RAPD 结果的分析方法}

种下类群包括亚种、品种 (系)、地理群体等。遗传分析的目的在于了解类群的遗传多样 性、鉴别类群、分析类群间差异大小和微进化关系等。多数作者采用两种方法对 RAPD 结果进 行处理并对上述问题进行探讨（Williams et al. ,1990;Nei \& Li ,1979;Welsh \& McClelland， 1990 ;陈永久等, 1997)。一是寻找类群的特有遗传标记, 据此可将群体进行鉴别; 另一种则是 基于遗传相似率的分析，包括相似率比较、分析遗传距离、聚类分析等。 
个体间之所以产生不同的 RAPD 扩增带, 是因为基因组 DNA 的突变、插入、缺失等引起 的,这些变异在群体中是以基因频率来表现的。对于群体而言,RAPD 结果上存在差异应该指 对应的位点上出现扩增带个体的比例上的差异, 可当作表型频率上的差异。当某位点的表型 频率变化到极端时, 就成为通常所说的单态位点、缺失位点。因此, 所谓可鉴别群体的遗传标 记, 正是不同群体表型频率不同的表现。基于这些考虑, 可以通过卡平方检验来分析群体在对 应位点上表型频率的差异。当使用大样本来研究群体间的差异时, 表型频率在统计意义上的 差异与遗传标记对区分群体具有同等重要的意义。

在群体遗传学中，根据基因频率、基因型频率来计算群体的多样性以及评价群体的分化程 度等已有较为成熟的方法。可以借鉴这些方法, 用 Shannon 指数来评价群体的表型多样性。 该指数从信息论的角度导出，用以测量信息序 (order) 或无序 (disorder) 的含量, 即不确定性的 含量 (钟扬等,1990) 其计算公式为 $h=-\sum P_{i} \log _{2} P_{i}$,式中 $P_{i}$ 为出现信息源中第 $i$ 个字符的 概率，必须满足 $0 \leqslant P_{i} \leqslant 1$ 和 $\sum P_{i}=1$ 两个条件, 这里指群体在一个位点上有带、无带的表型频 率 $; h$ 为信息源的信息量, 即 Shannon 多样性指数，单位比特 (bit) ,这里表示群体在该位点上表 型的多样性，或称多样度。当 $P_{1}=P_{2}=\cdots=P_{i}$ 时，信息量达到最大值;而当 $P_{i}=0$ 或 $P_{i}=1$ 时 ,规定信息量为 0 。对于 RAPD 结果“，符号”只有两种，即有带和无带两种表型。当群体在 某位点上有带和无带个体各占 $50 \%$ 时，信息量最大，多样性最高;而当该位点为单态或缺失 时, 多样性为 0 。因此, 当所研究的位点有一个以上的多态位点时, 可以用所有位点多样度的 平均值 $(H)$ 来表述群体 RAPD 扩增的多样性。

Shannon 指数还可以用来估测遗传多样性在群体内和群体间的分布, 即估测群体的遗传 分化程度。方法如下: 分别求出各类群的平均多样度, 然后求得几个类群多样度的平均值 $H_{p o p}$ 将要研究的若干个群体合并组成总群体, 并据总群体的表型频率求出总群体的平均多样 度 $H_{T}$; 以下式计算群体分化指数: $G s t=\left(H_{T}-H_{p o p}\right) / H_{T}$, 该值在 0 和 1 之间, 表示群体间多样度 占总多样度的比例，它反映了群体的遗传分化程度。

由于 RAPD 技术能够快速、简便地检测大量基因组 DNA 的遗传变异, 只要采用恰当的分 析方法, RAPD 技术不仅可用于鉴定品系、群体, 探讨进化关系, 还可在探讨群体分化等群体遗 传学其他方面发挥重要作用。

致谢＼cjkstart感谢中国科学院水生生物研究所淡水鱼类生物进化和生物地理实验室提供实验条 件。

\section{参 考 文 献}

常剑波, 王剑伟, 曹文宣, 1995。稀有】鲫胚胎发育研究. 水生生物学报, 19(2) 97 103

陈永久，王文，杨跃雄，宿兵，张亚平，熊绿芸，何照范，舒畅，扬大荣，1997。冬虫夏草(Cordyceps sinensis)的随机

扩增多态 DNA 及其遗传分化. 遗传学报 24(5):410 416

何舜平，王伟，陈宜瑜，2000。低等鲤科鱼类 RAPD 分析及系统发育研究. 水生生物学报 24(2) :101 106 何舜平, 汪亚平, 陈宜瑜, 1997. 五种鲤科鱼类的 RAPD 分析兼论稀有 鲫的系统位置. 水生生物学报, 21

(3) $262 \sim 267$

汪亚平,王剑伟,1997. 稀有】鲫同工酶研究. 鱼类学论文集(第六辑). 北京 科学出版社, 92 97

王剑伟,1992. 稀有鲫的繁殖生物学. 水生生物学报, 16(3):165 174

王剑伟, 1999. 稀有鲫产卵频次和卵子发育的研究. 水生生物学报 23(2) :161 166

王剑伟, 曹文宣, 1997. 稀有鲫与鱼类实验动物. 鱼类学论文集(第六辑). 北京 科学出版社, 144 152

王剑伟, 宋天祥, 曹文宣, 1998. 室养条件下稀有】鲫的胚后发育与幼鱼生长的研究. 水生生物学报, 22 (2):

$128 \sim 134$ 
肖武汉, 吴春花,宿兵, 张亚平, 崔桂华, 1997. 福尔马林固定云南鲖 DNA 提取及其细胞色素 $\mathrm{b}$ 基因序列分析. 动物学研究, 18(3) $242,252,258,284$

恽锐, 钟敏, 王洪新, 魏伟, 胡志昂, 钱迎倩, 1998. 北京东灵山辽东栎种群 DNA 多样性的研究. 植物学报, $\mathbf{4 0}$ (2) : : 169 175

钟扬,陈家宽,黄德世 (编著) ,1990. 数量分类的方法与程序. 武汉 武汉大学出版社

Apostol B L, Black IV W C, Miller B R, Reiter P, Beaty B J , 1993. Estimation of the number of full sibling families at an oviposition site using RAPD_PCR markers : applications to the mosquito Aedes aegypti. Theoretical and Appllied Genetics, $86991 \sim 1000$

Nei M , Li W H , 1979. Mathematical model for studying genetic variation in terms of restriction endonucleases. Proceedings of the National Academy of Sciences USA, 75(10) $5269 \sim 5273$

Sambrook J , Fritsch E F , Maniatic T (ed. ) , 金冬雁, 黎孟枫(译) ,1995. 分子克隆实验指南 (第二版). 北京： 科学出版社

Welsh J , McClelland M , 1990. Fingerprinting genomes using PCR with arbitrary primers. Nucleic Acids Research, $18: 7213 \sim 7218$

Welsh J , Peterson C, McClelland M , 1991. Polymorphism generated by arbitrarily primed PCR in the mouse : application to strain identification and genetic mapping. Nucleic Acids Research, $19303 \sim 306$

Williams J G K , Kubelik A R, Livak K J , Rafalski J A , Tingey S V , 1990. DNA polymorphisms amplified by arbitrary primers are useful as genetic markers. Nucleic Acids Research, 18(22): $6531 \sim 6535$

(本文由何舜平编委推荐, 责任编辑 :时意专) 